\title{
The Role of MuSK in Synapse Formation and Neuromuscular Disease
}

\author{
Steven J. Burden, Norihiro Yumoto, and Wei Zhang \\ Molecular Neurobiology Program, Helen L. and Martin S. Kimmel Center for Biology and Medicine at \\ the Skirball Institute of Biomolecular Medicine, NYU Medical School, New York, New York 10016 \\ Correspondence: burden@saturn.med.nyu.edu
}

\begin{abstract}
Muscle-specific kinase (MuSK) is essential for each step in neuromuscular synapse formation. Before innervation, MuSK initiates postsynaptic differentiation, priming the muscle for synapse formation. Approaching motor axons recognize the primed, or prepatterned, region of muscle, causing motor axons to stop growing and differentiate into specialized nerve terminals. MuSK controls presynaptic differentiation by causing the clustering of Lrp4, which functions as a direct retrograde signal for presynaptic differentiation. Developing synapses are stabilized by neuronal Agrin, which is released by motor nerve terminals and binds to Lrp4, a member of the low-density lipoprotein receptor family, stimulating further association between Lrp4 and MuSK and increasing MuSK kinase activity. In addition, MuSK phosphorylation is stimulated by an inside-out ligand, docking protein-7 (Dok-7), which is recruited to tyrosine-phosphorylated MuSK and increases MuSK kinase activity. Mutations in MuSK and in genes that function in the MuSK signaling pathway, including Dok-7, cause congenital myasthenia, and autoantibodies to MuSK, Lrp4, and acetylcholine receptors are responsible for myasthenia gravis.
\end{abstract}

\begin{abstract}
$M$ uscle-specific kinase (MuSK) is a singlepass transmembrane protein that has a critical role in signaling between motor neurons and skeletal muscle. MuSK is expressed in skeletal muscle cells, and once activated, MuSK stimulates pathways that (1) cluster and anchor acetylcholine receptors (AChRs) and additional muscle proteins that are critical for synaptic transmission, (2) enhance transcription of genes encoding synaptic proteins in muscle "synaptic nuclei," and (3) promote the production of retrograde signals that stimulate presynaptic differentiation. In the absence of MuSK, neuromuscular synapses fail to form (DeChiara et al.
\end{abstract}

1996). Mutations that impair MuSK kinase activity or signaling steps downstream from MuSK cause congenital myasthenia, characterized by structurally and functionally defective synapses, leading to muscle weakness and fatigue (Beeson et al. 2006; Muller et al. 2007; Selcen et al. 2008). In addition, autoantibodies to MuSK, as well as autoantibodies to AChRs and Lrp4, which forms a complex with MuSK (see below), are responsible for autoimmune myasthenia gravis (MG).

Here we describe the structure of MuSK; the mechanisms by which Agrin, a motor neuronderived signal, stimulates MuSK; the role of Lrp4, the receptor for Agrin and ligand for MuSK, in

Editors: Joseph Schlessinger and Mark A. Lemmon

Additional Perspectives on Signaling by Receptor Tyrosine Kinases available at www.cshperspectives.org

Copyright (C) 2013 Cold Spring Harbor Laboratory Press; all rights reserved; doi: 10.1101/cshperspect.a009167

Cite this article as Cold Spring Harb Perspect Biol 2013;5:a009167 
S.J. Burden et al.

activating MuSK; and the signaling steps downstream from MuSK.

\section{MUSK IS ESSENTIAL FOR SYNAPSE FORMATION}

MuSK, which is expressed by skeletal muscle and not by motor neurons, is essential for the formation and maintenance of neuromuscular synapses (Jennings et al. 1993; Valenzuela et al. 1995; DeChiara et al. 1996). MuSK acts in two phases of synapse formation: (1) prepatterning muscle in the prospective synaptic region before innervation, and (2) responding to neuronal Agrin to form and stabilize synapses.

Before motor innervation, AChRs and additional synaptic proteins are preferentially expressed and clustered in the central, prospective synaptic region of muscle in a manner that is independent of nerve-derived signals but dependent on MuSK (Yang et al. 2000, 2001; Lin et al. 2001; Arber et al. 2002; Burden 2011). This muscle prepatterning has a role in directing where motor axons grow and form synapses (Fig. 1) (Kim and Burden 2008). In the absence of MuSK, AChRs are expressed uniformly in muscle, and motor axons reach the muscle but fail to terminate and instead grow aimlessly without forming synapses (Fig. 2) (DeChiara et al. 1996). Muscle prepatterning is also dependent on Lrp4, which associates with MuSK and is sufficient to activate MuSK in nonmuscle cells (Weatherbee et al. 2006; Kim et al. 2008; Zhang et al. 2008) (see below).

$\mathrm{MuSK}$ itself is prepatterned in muscle (Kim and Burden 2008), but how MuSK expression and activity become confined to the prospective synaptic region is poorly understood. One idea suggests that prepatterning depends on (1) early expression of Lrp4 and MuSK in developing myotubes; (2) activation of MuSK by Lrp4; (3) positive-feedback mechanisms, dependent on MuSK activity, which enhance MuSK and Lrp4 expression in muscle nuclei that are near the site of MuSK activation; (4) clustering of Lrp4 and MuSK at sites where MuSK is activated; and (5) the orderly pattern of muscle growth, achieved by sequential fusion of myoblasts at the ends of growing myotubes (Kim and Burden 2008). The mechanisms that hinder MuSK
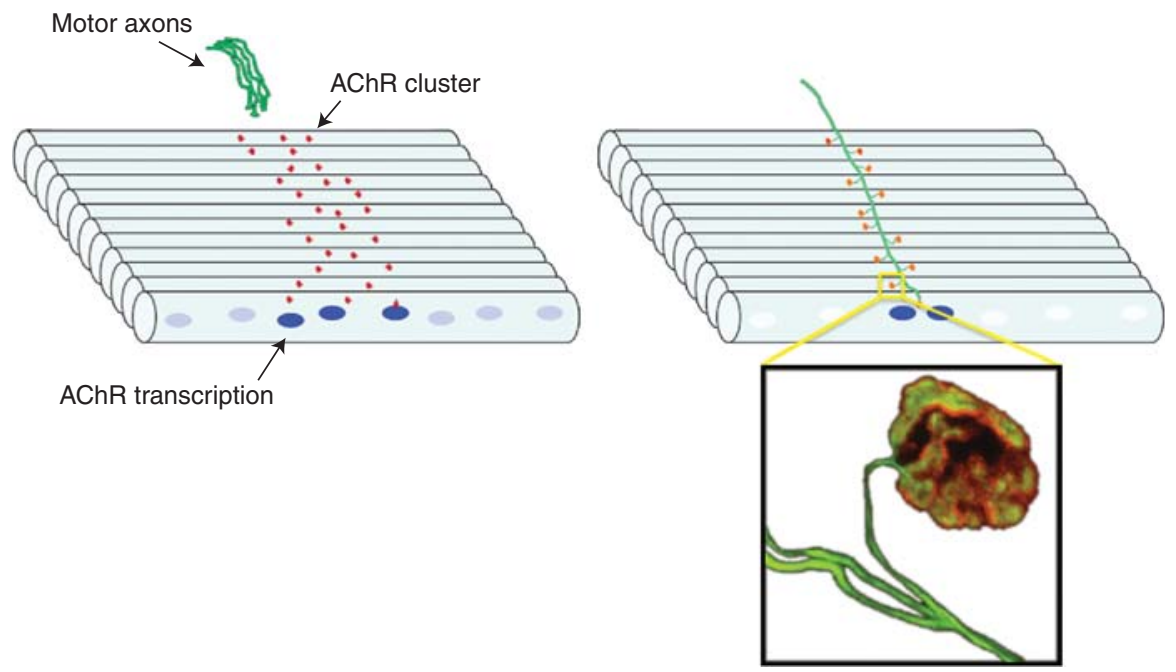

Figure 1. During development, motor axons (green) approach muscle in which AChR gene expression (blue) and clustering of AChRs (red) is enhanced in the prospective synaptic region of muscle. Motor axons form synapses in the prepatterned region of muscle. Motor axons release Agrin, which binds Lrp4 and stimulates MuSK, enhancing postsynaptic differentiation; and ACh, which depolarizes muscle, suppressing AChR expression. The combination of the two antagonist signals sharpens the prepattern so that AChR expression is restricted to synaptic sites. 


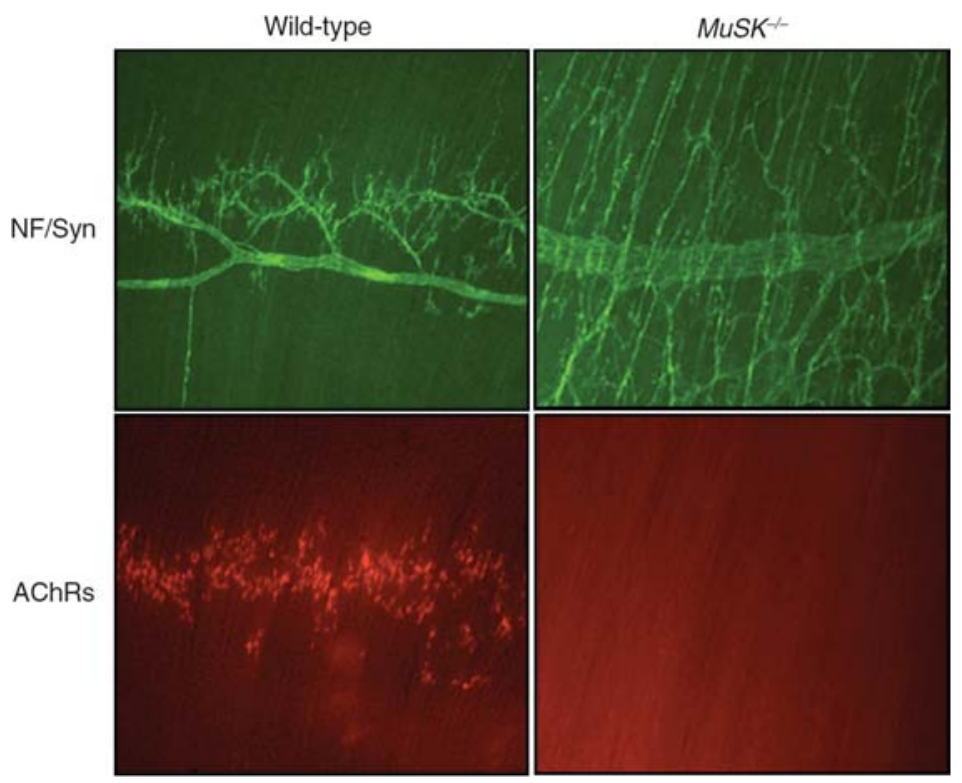

Figure 2. In the absence of MuSK, AChRs (red) fail to cluster and motor axons (green) fail to stop and differentiate. Motor axons and nerve terminals were stained with antibodies to neurofilament (NF) and synaptophysin (Syn), respectively.

activation and AChR clustering in peripheral regions of muscle are not fully understood.

Additional muscle-derived ligands may regulate MuSK activity; notably, Wnt11R, which binds to the Frizzled-like domain in MuSK (see below), can stimulate prepatterning in adaxial muscles of zebrafish (Jing et al. 2009). Further, murine Wnt9a, as well as Wnt11, can bind MuSK and stimulate clustering of AChRs, in a manner that depends on Lrp4 (Zhang et al. 2012a), suggesting that Wnts may serve as muscle-derived ligands that activate MuSK and stimulate muscle prepatterning. Because the hydrophobic pocket in Frizzled, which binds the lipid moiety attached to Wnts (Janda et al. 2012), is not present in the MuSK Frizzled-like domain (Stiegler et al. 2009) (see below), Wnts apparently bind MuSK in a manner that is distinct from the way that Wnts bind to Frizzled. It will be interesting to learn whether the MuSK Frizzled-like domain, which is not required for Agrin to stimulate AChR clustering (Zhou et al. 1999), is required for muscle prepatterning and whether Wnt signaling is required for muscle prepatterning and synapse formation in vivo in mammals.
Motor axons branch and arborize in a stereotyped manner within the prepatterned, central region of muscle, forming synapses in a narrow band adjacent to the main intramuscular nerve. Agrin, released from the tips of motor axons, binds directly to Lrp4, which stimulates further association between Lrp4 and MuSK and substantially increases MuSK phosphorylation (Figs. 3 and 4) (Kim et al. 2008; Zhang et al. 2008, 2011). Once MuSK is phosphorylated, signaling downstream from MuSK leads to clustering of Lrp4 and MuSK (see below), as well as clustering of AChRs and synapse-specific gene expression. Clustered Lrp4 signals in turn to motor axons, stimulating their differentiation (Yumoto et al. 2012). As such, Lrp4 functions bidirectionally, as it not only responds to Agrin, stimulating MuSK and postsynaptic differentiation, but also, once clustered as part of the program for postsynaptic differentiation, signals in a retrograde manner to motor neurons to stimulate presynaptic differentiation. Thus, Lrp4 coordinates synaptic differentiation (Yumoto et al. 2012).

MuSK is not only required to form neuromuscular synapses during embryogenesis but is 
S.J. Burden et al.

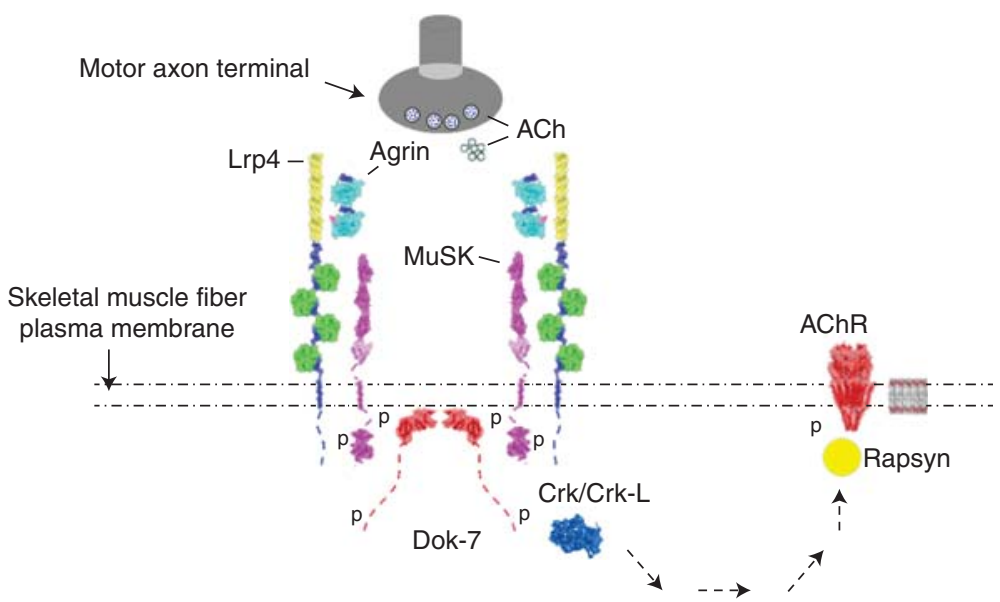

Figure 3. Motor axons release Agrin and ACh. Agrin binds to Lrp4, which stimulates association between Lrp4 and MuSK and MuSK phosphorylation. Once phosphorylated in the juxtamembrane region, MuSK recruits Dok-7, which forms a dimer and stabilizes a dimer of MuSK. Tyrosine phosphorylation of Dok-7 stimulates recruitment of Crk/Crk-L, which activates a poorly understood signaling pathway that leads to the anchoring of Rapsyn and AChRs at sites where MuSK is activated.

also essential to maintain neuromuscular synapses in adults. Reducing MuSK expression in adult mice, either by RNA interference or by conditional gene inactivation, leads to disassembly of the postsynaptic membrane and destabilization of synapses (Kong et al. 2004; Hesser et al. 2006). Consistent with the idea that MuSK has a critical role at adult synapses, $\sim 15 \%$ of patients with MG have autoantibodies to MuSK (Vincent and Leite 2005) (see below).

\section{MUSK STRUCTURE}

\section{The MuSK Extracellular Region}

The extracellular region of MuSK contains three immunoglobulin (Ig)-like domains and a Frizzled-like domain (Fig. 4A). In fish, avians, amphibians, and reptiles, a kringle domain is additionally present in the extracellular region (Jennings et al. 1993; Fu et al. 1999; Ip et al. 2000). The role of this kringle domain is not known, but its presence suggests an evolutionary relationship to Ror1 and Ror2, mammalian kringle-containing kinases, as well as CAM-1 in Caenorhabditis elegans and Dnrk and Dror in Drosophila (Wilson et al. 1993; Francis et al. 2005; Green et al. 2008).
The first Ig-like (Ig1) domain in MuSK forms a homodimer, and mutation of the hydrophobic dimer interface in Ig1, as well as deletion of the entire Ig1 domain, prevents Agrin from stimulating MuSK activation (Zhou et al. 1999; Stiegler et al. 2006). These findings indicate that MuSK activation requires MuSK dimerization, mediated, at least in part, by the first Ig-like domain. The Ig1 domain is also involved in binding Lrp4, and mutation of a solventexposed residue in the Ig1 domain, opposite to the hydrophobic dimer interface, prevents association between MuSK and Lrp4 and blocks Agrin from stimulating MuSK (Stiegler et al. 2006; Zhang et al. 2011). These findings raise the possibility that binding of MuSK to Lrp4 may orient the hydrophobic dimer interface to promote the formation or stability of a MuSK homodimer.

The Frizzled-like domain is not required for Agrin to activate MuSK, but it remains possible that the Frizzled-like domain engages alternative ligands, such as Wnts, that can activate MuSK (Zhou et al. 1999; Jing et al. 2009).

\section{The MuSK Intracellular Region}

The intracellular region of MuSK includes a 52amino-acid juxtamembrane region; a typical 
A

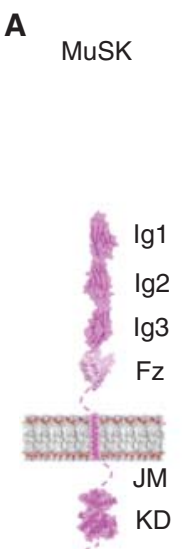

B

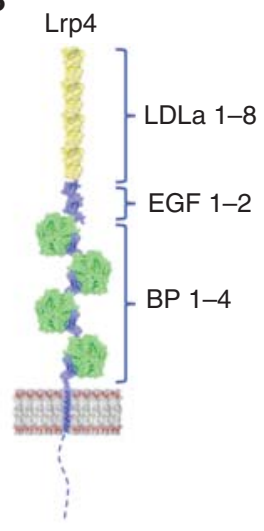

Figure 4. The domain organization of MuSK and Lrp4. (A) The extracellular region of MuSK contains three Ig-like domains and a Frizzled-like domain (Fz). The intracellular region of MuSK contains a juxtamembrane region (JM), a kinase domain (KD), and a short cytoplasmic tail. The crystal structures of the first two Ig-like domains (Stiegler et al. 2006), the Frizzled-like domain (Stiegler et al. 2009), and the intracellular region (Till et al. 2002) have been determined, but their arrangement in the context of the full kinase is not known and is depicted here only for illustrative purposes. $(B)$ The extracellular region of Lrp4 contains eight LDLa repeats, two EGF-like domains, and four BP domains with embedded EGFlike domains. The crystal structure of the first BP domain in Lrp4 has been determined (Zong et al. 2012), whereas the structures of all other domains in Lrp4 have been inferred from the structures of these domains in other Lrp family members (Rudenko et al. 2002). The arrangement of these domains in the context of the full receptor is not known and is shown only for illustrative purposes.

kinase domain, containing three tyrosine residues within the activation loop; and an eightamino-acid carboxy-terminal sequence (Jennings et al. 1993; Valenzuela et al. 1995; Till et al. 2002). Four tyrosine residues, the three in the activation loop and one in the juxtamembrane region, are the major sites for tyrosine phosphorylation in MuSK (Watty et al. 2000; Till et al. 2002).

\section{Lrp4 STRUCTURE AND FUNCTION}

The extracellular region of Lrp4 contains eight low-density lipoprotein receptor domain class A (LDLa) repeats, two epidermal growth factor

MuSK at Synapses and in Disease

(EGF)-like domains, and four $\beta$-propeller (BP) domains, which are joined to an EGF-like domain (Fig. 4B). The last few LDLa repeats together with the first BP domain in Lrp4 are required for maximal binding of neural Agrin (Zhang et al. 2011), whereas the second and third BP domains in Lrp4 are required for Lrp4 to bind MuSK and for Agrin to stimulate MuSK phosphorylation (Zhang et al. 2011). Binding of Agrin to Lrp4 stimulates association between Lrp4 and MuSK, indicating that Agrin causes structural changes in Lrp4 to promote association with MuSK and stimulate MuSK kinase activity (Zhang et al. 2011). Unfortunately, isolation of the extracellular region of Lrp4 has proved difficult, impeding structural studies. A crystal structure of a complex between the first BP domain of Lrp4 and the carboxy-terminal region of Agrin, mini-Agrin, a 21-kDa protein containing the third laminin G-like domain, reveals that residues in the neuronal-specific insert of Agrin interact with the first BP domain in Lrp4. The structure also suggests a 2:2 Agrin/ Lrp4 complex, mediated by an Agrin dimer, which could recruit two MuSK molecules into the complex for trans-phosphorylation of MuSK and initiation of downstream signaling (Zong et al. 2012).

Mini-Agrin, analyzed in the crystal structure, activates MuSK 100-fold less efficiently than recombinant forms of neural Agrin (e.g., $50 \mathrm{kDa}$ ) containing two laminin G-like domains and the last of four EGF-like domains (Gesemann et al. 1995). Although mini-Agrin binds the first BP domain in Lrp4 (Zong et al. 2012), the last few LDLa repeats in Lrp4 are required for maximal binding between Lrp4 and the $50-\mathrm{kDa}$ form of neural Agrin (Zhang et al. 2011). Thus, these LDLa repeats may contact the second laminin G-like domain or the last EGF-like domain in neural Agrin and confer the $\sim 100$-fold-greater activity of the larger forms of Agrin.

Although Agrin causes Lrp4 to adopt a configuration that is competent to bind and activate MuSK (Zhang et al. 2011), Lrp4 can bind and activate MuSK independently of Agrin (Kim et al. 2008; Zhang et al. 2011). The ability of Lrp4 to activate MuSK likely underlies the role 
S.J. Burden et al.

of Lrp4 in muscle prepatterning. Thus, Lrp4 may be in a dynamic equilibrium, and Agrin may shift this equilibrium to favor a conformation that binds MuSK.

Because Lrp4 self-associates, independently of Agrin, a preformed oligomer of Lrp4 may serve as a template to recruit MuSK and facilitate the formation of a MuSK dimer, promoting MuSK trans-phosphorylation (Kim et al. 2008; Zhang et al. 2011). The formation or stability of MuSK dimers may also be enhanced by sequences that promote homodimerization of MuSK, as structural studies reveal that a hydrophobic surface in the first Ig-like domain in MuSK mediates formation of a homodimer, and mutation of this interface prevents Agrin from activating MuSK (Stiegler et al. 2006).

Because Agrin binds Lrp4 rather than MuSK, MuSK is not a traditional receptor tyrosine kinase. Because Lrp4 binds and stimulates MuSK kinase activity, whereas Agrin promotes association between Lrp4 and MuSK, Lrp4 appears to function as a ligand for MuSK, whereas Agrin behaves as an allosteric regulator. In this sense, MuSK resembles Ret and ErbB2, tyrosine kinases that associate with partner proteins, GDNFR and ErbB1, ErbB3, or ErbB4, respectively, which serve as receptors for activating ligands (Zhang et al. 2011).

\section{SIGNALING DOWNSTREAM FROM MUSK}

Once the juxtamembrane tyrosine, Y553, becomes phosphorylated, MuSK recruits docking protein-7 (Dok-7), a noncatalytic adapter protein (Fig. 3) (Okada et al. 2006). Dok-7 contains amino-terminal pleckstrin homology and phosphotyrosine-binding domains, required to bind pY553 MuSK, as well as a carboxy-terminal domain (Okada et al. 2006). In the absence of Dok7 , as in the absence of MuSK, neuromuscular synapses fail to form (Okada et al. 2006). Further, hypomorphic mutations in human Dok-7, which impair Dok-7 function, cause congenital myasthenia (Beeson et al. 2006) (see below).

Once recruited to MuSK, Dok-7 stimulates further MuSK phosphorylation and increases MuSK kinase activity (Okada et al. 2006; Inoue et al. 2009; Bergamin et al. 2010), showing that
Dok-7 functions, at least in part, as an insideout ligand for MuSK. Consistent with this idea, the pleckstrin homology and phosphotyrosinebinding domains of Dok-7 form a dimer, which binds MuSK, promotes the formation of MuSK dimers, and stimulates MuSK phosphorylation in vitro (Bergamin et al. 2010). Moreover, certain disease-causing mutations in the phosphotyrosine binding domain of human Dok-7 impair Dok-7 dimerization, showing that Dok-7 dimerization is critical to activate MuSK in vivo (Bergamin et al. 2010).

A majority of patients with Dok-7 congenital myasthenia carry mutations that truncate the carboxy-terminal domain, indicating that the carboxy-terminal domain has an important function, separate from the role of the aminoterminal domain in stimulating MuSK kinase activity (Muller et al. 2007). Agrin stimulates phosphorylation of two tyrosine residues in the carboxy-terminal domain of Dok-7, which leads to recruitment of two adapter proteins, Crk and Crk-L (Hallock et al. 2010). Muscleselective inactivation of Crk and Crk-L causes defects in neuromuscular synapses that are virtually identical to the aberrant synapses found in humans with Dok-7 congenital myasthenia caused by mutations that truncate Dok-7 and prevent phosphorylation of Dok-7 at these two sites (Hallock et al. 2010). Thus, Dok-7 not only stimulates MuSK phosphorylation but also functions downstream from MuSK and transduces MuSK activation to synaptic differentiation. The molecules that are recruited to the Crk/Crk-L/Dok-7/MuSK complex and function in postsynaptic differentiation have not been identified. Ultimately, Dok-7 recruitment and phosphorylation lead to the redistribution and anchoring of critical proteins to the postsynaptic membrane (Fig. 3). This pathway requires Rac, Rho, Actin, and Rapsyn, but additional players are clearly involved (Dai et al. 2000; Weston et al. 2000, 2003; Ramarao et al. 2001; Burden 2011). Rapsyn, an intracellular, peripheral membrane protein, binds directly to AChRs (Neubig et al. 1979; Burden et al. 1983), but how MuSK activation and Dok-7 recruitment regulate the distribution and anchoring of Rapsyn is not currently understood. In ad- 
dition, MuSK and Dok-7, but not Rapsyn, are required for synapse-specific gene expression, which appears to involve activation of JNK and Ets-domain-containing proteins (Gautam et al. 1995; Fromm and Burden 1998; Schaeffer et al. 1998; Si et al. 1999; Weston et al. 2000; Hippenmeyer et al. 2007). Thus, the pathways downstream from Dok-7 ultimately diverge, as the posttranslational pathway for anchoring postsynaptic proteins requires Rapsyn, whereas the transcriptional pathway for stimulating "synaptic genes" is Rapsyn-independent.

\section{AUTOIMMUNE MG AND MYASTHENIA}

Autoantibodies to the AChR, MuSK, or Lrp4 are responsible for $\mathrm{MG}$, an autoimmune disease with a prevalence of $1-2$ in 10,000 (Kalb et al. 2002; Murai et al. 2011). Intermittent muscle weakness, which worsens after activity, is the hallmark feature of MG. Autoantibodies to the AChR are responsible for most $(\sim 80 \%)$ cases of MG. A preponderance of these antibodies are directed to the main immunogenic region in the AChR $\alpha$ subunit, which can block AChR function, increase AChR turnover, and/or stimulate complement-mediated damage (Fig. 5).
Anti-AChR MG is treated by plasmapheresis, systemic immunosuppression, and anticholinesterases, which prolong the action of acetylcholine $(\mathrm{ACh})$ and thereby enhance synaptic transmission.

Approximately $15 \%$ of patients with $\mathrm{MG}$ have autoantibodies to MuSK, although the percentage varies among different ethnic groups (Evoli et al. 2008; Vincent et al. 2008). Muscle weakness in anti-MuSK MG can be severe, requiring respiratory assistance. Moreover, the disease is recalcitrant to treatments, such as acetylcholinesterase inhibitors, which effectively manage MG caused by autoantibodies to the AChR (Farrugia and Vincent 2010). As such, current treatment for anti-MuSK MG relies on systemic immunosuppression. Unlike autoantibodies to the AChR, the disease-causing autoantibodies to MuSK are largely IgG4, which are functionally monovalent and do not engage complement, indicating that these antibodies interfere with MuSK function rather than causing cell damage (Niks et al. 2008; Klooster et al. 2012). It will be important to learn whether these antibodies obstruct association of MuSK with Lrp4 or interfere with the formation of MuSK dimers.

Congenital myasthenia

\begin{tabular}{|c|c|}
\hline $\begin{array}{c}\text { Agrin } \\
\text { MuSK } \\
\text { Dok-7 } \\
\text { Rapsyn }\end{array}$ & $\begin{array}{l}\text { Mutations impair postsynaptic differentiation, } \\
\text { compromising synaptic transmission and leading } \\
\text { to muscle weakness and fatigue. }\end{array}$ \\
\hline $\begin{array}{c}\text { AChR } \\
\text { AChE } \\
\text { ChAT } \\
\text { Nav1.4 }\end{array}$ & $\begin{array}{l}\text { Mutations impair synaptic transmission, leading } \\
\text { to muscle weakness and fatigue. }\end{array}$ \\
\hline & Myasthenia gravis \\
\hline $\begin{array}{l}\text { Autoantibodies } \\
\text { to AChRs }\end{array}$ & $\begin{array}{l}\text { Inhibit binding of ACh, stimulate accelerated } \\
\text { degradation of AChRs and cause structural } \\
\text { disorganization of the synapse. }\end{array}$ \\
\hline $\begin{array}{l}\text { Autoantibodies } \\
\text { to MuSK or Lrp4 }\end{array}$ & $\begin{array}{l}\text { Autoantibodies to MuSK are IgG4, suggesting } \\
\text { that they interfere with MuSK function, } \\
\text { rather than recruiting complement. }\end{array}$ \\
\hline
\end{tabular}

Figure 5. Neuromuscular diseases: autoimmune myasthenia gravis and congenital myasthenia. 
S.J. Burden et al.

A smaller percentage $(0.5 \%-5 \%)$ of $\mathrm{MG}$ patients have autoantibodies to Lrp4 (Higuchi et al. 2011; Pevzner et al. 2012; Zhang et al. 2012b). The clinical and pathological features of anti-Lrp4 MG have not been well defined. Autoantibodies to Lrp4 inhibit binding between Lrp4 and Agrin (Higuchi et al. 2011), but may also interfere with synaptic function by preventing oligomerization of Lrp4 or blocking association between Lrp4 and MuSK.

In anti-AChR MG, reduced expression of AChRs in the postsynaptic membrane leads to a decreased responsiveness of the postsynaptic membrane to ACh.

This postsynaptic change is accompanied by an increase in ACh release from motor nerve terminals, indicative of a compensatory mechanism that enhances synaptic transmission. In anti-MuSK MG, the postsynaptic membrane is likewise less responsive to $\mathrm{ACh}$, but $\mathrm{ACh}$ release is not enhanced (Klooster et al. 2012). Together, these data suggest that the compensatory mechanism, initiated by a decrease in postsynaptic responsiveness and leading to an increase in transmitter release, requires MuSK.

Congenital myasthenia is caused by mutations in genes that are essential for the formation or function of the synapse (Fig. 5) (Engel et al. 2003). Congenital myasthenia is less common than MG and has an estimated prevalence of 1 in 500,000 in Europe. Mutations in $A C h R$ subunit genes, Rapsyn, and Dok-7 are major causes of congenital myasthenia. Mutations in $M u S K$ are a less common cause for congenital myasthenia. One patient, who is transheterozygous for two mutant MuSK alleles, suffers from congenital myasthenia, typified by highly fatigable muscle weakness (Chevessier et al. 2004). One allele is an early-frameshift null mutation, whereas the other allele truncates the kinase domain.

\section{MUSK EXPRESSION AND FUNCTION OUTSIDE THE NEUROMUSCULAR SYNAPSE}

Mice and zebrafish deficient in MuSK have defects in neural crest migration (Banerjee et al. 2011). In the absence of MuSK, streaming neural crest cells, which are normally confined to the central region of each somite, are dispersed and present throughout the somite. These data indicate that muscle cells provide guidance clues, which are dependent on MuSK activity, not only to motor axons but also to neural crest cells, thereby confining their movement to the prepatterned zone of muscle.

Skeletal muscle is the major site for $M u S K$ expression. MuSK expression is regulated during myogenesis, as MuSK, like Lrp4 and Dok-7, is expressed in myotubes but not in myoblasts (Valenzuela et al. 1995; Okada et al. 2006; Kim et al. 2008). In adult muscle MuSK expression is largely restricted to the synaptic nuclei of muscle (Kim and Burden 2008), which constitute $\sim 1 \%$ of total myofiber nuclei (Merlie and Sanes 1985; Burden 1993).

MuSK is expressed in the central nervous system, where it is concentrated at excitatory synapses, marked by coexpression of PSD-95 and Agrin (Garcia-Osta et al. 2006; Ksiazek et al. 2007). MuSK expression is evident in the hippocampus, olfactory bulb, cerebellum, and cortex (see http://www.brainatlas.org). The role of MuSK in the central nervous system is poorly understood, although MuSK has been reported to have a role in memory consolidation (Garcia-Osta et al. 2006).

Further, MuSK RNA expression is detected in the neural tube of Xenopus tadpoles and in the nervous system of developing and adult chickens (Fu et al. 1999; Ip et al. 2000). Moreover, MuSK is expressed transiently in the developing liver of chick and rat and in the eye vesicles, spleen, and lung of Xenopus (Fu et al. 1999). The role for MuSK in nonmuscle cells is not currently understood.

\section{CONCLUSIONS}

Studies over the past 20 years have revealed that MuSK is a master regulatory kinase that is essential for each step in neuromuscular synapse formation. In the future we are likely to learn (1) how Agrin binding to Lrp4 alters the conformation of Lrp4 to promote association between Lrp4 and MuSK, (2) how the formation of a complex between Lrp4 and MuSK stimulates MuSK kinase activity, (3) which molecules 
function downstream from Dok-7 and link recruitment of Crk/Crk-L to Rapsyn and to synapse-specific transcription, (4) how autoantibodies to MuSK cause MG, (5) whether increasing MuSK activity might be therapeutic for diseases in which the neuromuscular synapse is compromised, and (6) the functions of MuSK outside the neuromuscular synapse.

\section{REFERENCES}

Arber S, Burden SJ, Harris AJ. 2002. Patterning of skeletal muscle. Curr Opin Neurobiol 12: 100-103.

Banerjee S, Gordon L, Donn TM, Berti C, Moens CB, Burden SJ, Granato M. 2011. A novel role for MuSK and non-canonical Wnt signaling during segmental neural crest cell migration. Development 138: 3287-3296.

Beeson D, Higuchi O, Palace J, Cossins J, Spearman H, Maxwell S, Newsom-Davis J, Burke G, Fawcett P, Motomura M, et al. 2006. Dok-7 mutations underlie a neuromuscular junction synaptopathy. Science 313: 1975-1978.

Bergamin E, Hallock PT, Burden SJ, Hubbard SR. 2010. The cytoplasmic adaptor protein Dok7 activates the receptor tyrosine kinase MuSK via dimerization. Mol Cell 39: 100-109.

Burden SJ. 1993. Synapse-specific gene expression. Trends Genet 9: 12-16.

Burden SJ. 2011. SnapShot: Neuromuscular junction. Cell 144: 826-826.e1.

Burden SJ, DePalma RL, Gottesman GS. 1983. Crosslinking of proteins in acetylcholine receptor-rich membranes: Association between the $\beta$-subunit and the $43 \mathrm{kd}$ subsynaptic protein. Cell 35: 687-692.

Chevessier F, Faraut B, Ravel-Chapuis A, Richard P, Gaudon K, Bauche S, Prioleau C, Herbst R, Goillot E, Ioos C, et al. 2004. MUSK, a new target for mutations causing congenital myasthenic syndrome. Hum Mol Genet 13: 3229-3240.

Dai Z, Luo X, Xie H, Peng HB. 2000. The actin-driven movement and formation of acetylcholine receptor clusters. J Cell Biol 150: 1321-1334.

DeChiara TM, Bowen DC, Valenzuela DM, Simmons MV, Poueymirou WT, Thomas S, Kinetz E, Compton DL, Rojas E, Park JS, et al. 1996. The receptor tyrosine kinase MuSK is required for neuromuscular junction formation in vivo. Cell 85: 501-512.

Engel AG, Ohno K, Sine SM. 2003. Sleuthing molecular targets for neurological diseases at the neuromuscular junction. Nat Rev Neurosci 4: 339-352.

Evoli A, Bianchi MR, Riso R, Minicuci GM, Batocchi AP, Servidei S, Scuderi F, Bartoccioni E. 2008. Response to therapy in myasthenia gravis with anti-MuSK antibodies. Ann N Y Acad Sci 1132: 76-83.

Farrugia ME, Vincent A. 2010. Autoimmune mediated neuromuscular junction defects. Curr Opin Neurol 23: 489495.

Francis MM, Evans SP, Jensen M, Madsen DM, Mancuso J, Norman KR, Maricq AV. 2005. The Ror receptor tyrosine kinase CAM-1 is required for ACR-16-mediated synaptic transmission at the C. elegans neuromuscular junction. Neuron 46: 581-594.

Fromm L, Burden SJ. 1998. Synapse-specific and neuregulin-induced transcription require an Ets site that binds GABP $\alpha /$ GABP $\beta$. Genes Dev 12: 3074-3083.

Fu AK, Smith FD, Zhou H, Chu AH, Tsim KW, Peng BH, Ip NY. 1999. Xenopus muscle-specific kinase: Molecular cloning and prominent expression in neural tissues during early embryonic development. Eur J Neurosci 11: 373-382.

Garcia-Osta A, Tsokas P, Pollonini G, Landau EM, Blitzer R, Alberini CM. 2006. MuSK expressed in the brain mediates cholinergic responses, synaptic plasticity, and memory formation. J Neurosci 26: 7919-7932.

Gautam M, Noakes PG, Mudd J, Nichol M, Chu GC, Sanes JR, Merlie JP. 1995. Failure of postsynaptic specialization to develop at neuromuscular junctions of rapsyndeficient mice. Nature 377: 232-236.

Gesemann M, Denzer AJ, Ruegg MA. 1995. Acetylcholine receptor-aggregating activity of agrin isoforms and mapping of the active site. J Cell Biol 128: 625-636.

Green JL, Kuntz SG, Sternberg PW. 2008. Ror receptor tyrosine kinases: Orphans no more. Trends Cell Biol 18: 536-544.

Hallock PT, Xu CF, Park TJ, Neubert TA, Curran T, Burden SJ. 2010. Dok-7 regulates neuromuscular synapse formation by recruiting Crk and Crk-L. Genes Dev 24: 2451-2461.

Hesser BA, Henschel O, Witzemann V. 2006. Synapse disassembly and formation of new synapses in postnatal muscle upon conditional inactivation of MuSK. Mol Cell Neurosci 31: 470-480.

Higuchi O, Hamuro J, Motomura M, Yamanashi Y. 2011. Autoantibodies to low-density lipoprotein receptor-related protein 4 in myasthenia gravis. Ann Neurol 69: $418-422$.

Hippenmeyer S, Huber RM, Ladle DR, Murphy K, Arber S. 2007. ETS transcription factor Erm controls subsynaptic gene expression in skeletal muscles. Neuron 55: 726-740.

Inoue A, Setoguchi K, Matsubara Y, Okada K, Sato N, Iwakura Y, Higuchi O, Yamanashi Y. 2009. Dok-7 activates the muscle receptor kinase MuSK and shapes synapse formation. Sci Signal 2: ra7.

Ip FC, Glass DG, Gies DR, Cheung J, Lai KO, Fu AK, Yancopoulos GD, Ip NY. 2000. Cloning and characterization of muscle-specific kinase in chicken. Mol Cell Neurosci 16: 661-673.

Janda CY, Waghray D, Levin AM, Thomas C, Garcia KC. 2012. Structural basis of Wnt recognition by Frizzled. Science 337: 59-64.

Jennings CG, Dyer SM, Burden SJ. 1993. Muscle-specific trk-related receptor with a kringle domain defines a distinct class of receptor tyrosine kinases. Proc Natl Acad Sci 90: $2895-2899$.

Jing L, Lefebvre JL, Gordon LR, Granato M. 2009. Wnt signals organize synaptic prepattern and axon guidance through the zebrafish unplugged/MuSK receptor. Neuron 61: 721-733. 


\section{S.J. Burden et al.}

Kalb B, Matell G, Pirskanen R, Lambe M. 2002. Epidemiology of myasthenia gravis: A population-based study in Stockholm, Sweden. Neuroepidemiology 21: 221-225.

Kim N, Burden SJ. 2008. MuSK controls where motor axons grow and form synapses. Nature Neurosci 11: 19-27.

Kim N, Stiegler AL, Cameron TO, Hallock PT, Gomez AM, Huang JH, Hubbard SR, Dustin ML, Burden SJ. 2008. Lrp4 is a receptor for Agrin and forms a complex with MuSK. Cell 135: 334-342.

Klooster R, Plomp JJ, Huijbers MG, Niks EH, Straasheijm KR, Detmers FJ, Hermans PW, Sleijpen K, Verrips A, Losen M, et al. 2012. Muscle-specific kinase myasthenia gravis IgG4 autoantibodies cause severe neuromuscular junction dysfunction in mice. Brain 135: 1081-1101.

Kong XC, Barzaghi P, Ruegg MA. 2004. Inhibition of synapse assembly in mammalian muscle in vivo by RNA interference. $E M B O$ Rep 5: 183-188.

Ksiazek I, Burkhardt C, Lin S, Seddik R, Maj M, Bezakova G, Jucker M, Arber S, Caroni P, Sanes JR, et al. 2007. Synapse loss in cortex of agrin-deficient mice after genetic rescue of perinatal death. J Neurosci 27: 7183-7195.

Lin W, Burgess RW, Dominguez B, Pfaff SL, Sanes JR, Lee KF. 2001. Distinct roles of nerve and muscle in postsynaptic differentiation of the neuromuscular synapse. Nature 410: 1057-1064.

Merlie JP, Sanes JR. 1985. Concentration of acetylcholine receptor mRNA in synaptic regions of adult muscle fibres. Nature 317: 66-68.

Muller JS, Herczegfalvi A, Vilchez JJ, Colomer J, Bachinski LL, Mihaylova V, Santos M, Schara U, Deschauer M, Shevell M, et al. 2007. Phenotypical spectrum of DOK7 mutations in congenital myasthenic syndromes. Brain 130: 1497-1506.

Murai $\mathrm{H}$, Yamashita N, Watanabe M, Nomura Y, Motomura M, Yoshikawa H, Nakamura Y, Kawaguchi N, Onodera H, Araga S, et al. 2011. Characteristics of myasthenia gravis according to onset-age: Japanese nationwide survey. J Neurol Sci 305: 97-102.

Neubig RR, Krodel EK, Boyd ND, Cohen JB. 1979. Acetylcholine and local anesthetic binding to Torpedo nicotinic postsynaptic membranes after removal of nonreceptor peptides. Proc Natl Acad Sci 76: 690-694.

Niks EH, van Leeuwen Y, Leite MI, Dekker FW, Wintzen AR, Wirtz PW, Vincent A, van Tol MJ, Jol-van der Zijde CM, Verschuuren JJ. 2008. Clinical fluctuations in MuSK myasthenia gravis are related to antigen-specific IgG4 instead of IgG1. J Neuroimmunol 195: 151-156.

Okada K, Inoue A, Okada M, Murata Y, Kakuta S, Jigami T, Kubo S, Shiraishi H, Eguchi K, Motomura M, et al. 2006. The muscle protein Dok-7 is essential for neuromuscular synaptogenesis. Science 312: 1802-1805.

Pevzner A, Schoser B, Peters K, Cosma NC, Karakatsani A, Schalke B, Melms A, Kroger S. 2012. Anti-LRP4 autoantibodies in AChR- and MuSK-antibody-negative myasthenia gravis. J Neurol 259: 427-435.

Ramarao MK, Bianchetta MJ, Lanken J, Cohen JB. 2001. Role of rapsyn tetratricopeptide repeat and coiled-coil domains in self-association and nicotinic acetylcholine receptor clustering. J Biol Chem 276: 7475-7483.
Rudenko G, Henry L, Henderson K, Ichtchenko K, Brown MS, Goldstein JL, Deisenhofer J. 2002. Structure of the LDL receptor extracellular domain at endosomal pH. Science 298: 2353-2358.

Schaeffer L, Duclert N, Huchet-Dymanus M, Changeux JP. 1998. Implication of a multisubunit Ets-related transcription factor in synaptic expression of the nicotinic acetylcholine receptor. EMBO J 17: 3078-3090.

Selcen D, Milone M, Shen XM, Harper CM, Stans AA, Wieben ED, Engel AG. 2008. Dok-7 myasthenia: Phenotypic and molecular genetic studies in 16 patients. Ann Neurol 64: 71-87.

Si J, Wang Q, Mei L. 1999. Essential roles of c-JUN and cJUN N-terminal kinase (JNK) in neuregulin-increased expression of the acetylcholine receptor $\epsilon$-subunit. J Neurosci 19: 8498-8508.

Stiegler AL, Burden SJ, Hubbard SR. 2006. Crystal structure of the agrin-responsive immunoglobulin-like domains 1 and 2 of the receptor tyrosine kinase MuSK. J Mol Biol 364: 424-433.

Stiegler AL, Burden SJ, Hubbard SR. 2009. Crystal structure of the Frizzled-like cysteine-rich domain of the receptor tyrosine kinase MuSK. J Mol Biol 393: 1-9.

Till JH, Becerra M, Watty A, Lu Y, Ma Y, Neubert TA, Burden SJ, Hubbard SR. 2002. Crystal structure of the MuSK tyrosine kinase: Insights into receptor autoregulation. Structure 10: 1187-1196.

Valenzuela DM, Stitt TN, DiStefano PS, Rojas E, Mattsson K, Compton DL, Nunez L, Park JS, Stark JL, Gies DR, et al. 1995. Receptor tyrosine kinase specific for the skeletal muscle lineage: Expression in embryonic muscle, at the neuromuscular junction, and after injury. Neuron 15: $573-584$.

Vincent A, Leite MI. 2005. Neuromuscular junction autoimmune disease: Muscle specific kinase antibodies and treatments for myasthenia gravis. Curr Opin Neurol 18: 519-525.

Vincent A, Leite MI, Farrugia ME, Jacob S, Viegas S, Shiraishi H, Benveniste O, Morgan BP, Hilton-Jones D, Newsom-Davis J, et al. 2008. Myasthenia gravis seronegative for acetylcholine receptor antibodies. Ann NY Acad Sci 1132: 84-92.

Watty A, Neubauer G, Dreger M, Zimmer M, Wilm M, Burden SJ. 2000. The in vitro and in vivo phosphotyrosine map of activated MuSK. Proc Natl Acad Sci 97: 4585-4590.

Weatherbee SD, Anderson KV, Niswander LA. 2006. LDLreceptor-related protein 4 is crucial for formation of the neuromuscular junction. Development 133: 4993-5000.

Weston C, Yee B, Hod E, Prives J. 2000. Agrin-induced acetylcholine receptor clustering is mediated by the small guanosine triphosphatases Rac and Cdc42. J Cell Biol 150: $205-212$.

Weston C, Gordon C, Teressa G, Hod E, Ren XD, Prives J. 2003. Cooperative regulation by Rac and Rho of agrininduced acetylcholine receptor clustering in muscle cells. J Biol Chem 278: 6450-6455.

Wilson C, Goberdhan DC, Steller H. 1993. Dror, a potential neurotrophic receptor gene, encodes a Drosophila homo$\log$ of the vertebrate Ror family of Trk-related receptor tyrosine kinases. Proc Natl Acad Sci 90: 7109-7113. 
MuSK at Synapses and in Disease

Yang X, Li W, Prescott ED, Burden SJ, Wang JC. 2000. DNA topoisomerase II $\beta$ and neural development. Science 287: $131-134$.

Yang X, Arber S, William C, Li L, Tanabe Y, Jessell TM, Birchmeier C, Burden SJ. 2001. Patterning of muscle acetylcholine receptor gene expression in the absence of motor innervation. Neuron 30: 399-410.

Yumoto N, Kim N, Burden SJ. 2012. Lrp4 is a retrograde signal for presynaptic differentiation at neuromuscular synapses. Nature 489: 438-442.

Zhang B, Luo S, Wang Q, Suzuki T, Xiong WC, Mei L. 2008. LRP4 serves as a coreceptor of agrin. Neuron 60: 285-297.

Zhang W, Coldefy AS, Hubbard SR, Burden SJ. 2011. Agrin binds to the N-terminal region of Lrp4 and stimulates association between Lrp4 and the first Ig-like domain in MuSK. J Biol Chem 286: 40624-40630.
Zhang B, Liang C, Bates R, Yin Y, Xiong WC, Mei L. 2012a. Wnt proteins regulate acetylcholine receptor clustering in muscle cells. Mol Brain 5: 7.

Zhang B, Tzartos JS, Belimezi M, Ragheb S, Bealmear B, Lewis RA, Xiong WC, Lisak RP, Tzartos SJ, Mei L. 2012b. Autoantibodies to lipoprotein-related protein 4 in patients with double-seronegative myasthenia gravis. Arch Neurol 69: 445-451.

Zhou H, Glass DJ, Yancopoulos GD, Sanes JR. 1999. Distinct domains of MuSK mediate its abilities to induce and to associate with postsynaptic specializations. J Cell Biol 146: 1133-1146.

Zong Y, Zhang B, Gu S, Lee K, Zhou J, Yao G, Figueiredo D, Perry K, Mei L, Jin R. 2012. Structural basis of agrinLRP4-MuSK signaling. Genes Dev 26: 247-258. 


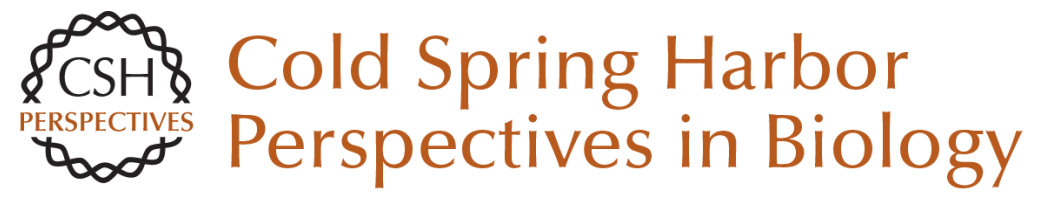

\section{The Role of MuSK in Synapse Formation and Neuromuscular Disease}

Steven J. Burden, Norihiro Yumoto and Wei Zhang

Cold Spring Harb Perspect Biol 2013; doi: 10.1101/cshperspect.a009167

Subject Collection Signaling by Receptor Tyrosine Kinases

CSF-1 Receptor Signaling in Myeloid Cells

E. Richard Stanley and Violeta Chitu

The EGFR Family: Not So Prototypical Receptor

Tyrosine Kinases

Mark A. Lemmon, Joseph Schlessinger and Kathryn M. Ferguson

Tie2 and Eph Receptor Tyrosine Kinase Activation and Signaling

William A. Barton, Annamarie C. Dalton, Tom C.M. Seegar, et al.

The Spatiotemporal Organization of ErbB

Receptors: Insights from Microscopy

Christopher C. Valley, Keith A. Lidke and Diane S. Lidke

Insulin Receptor Signaling in Normal and Insulin-Resistant States

Jérémie Boucher, André Kleinridders and C. Ronald Kahn

Central Role of RET in Thyroid Cancer Massimo Santoro and Francesca Carlomagno

Receptor Tyrosine Kinase-Mediated Angiogenesis Michael Jeltsch, Veli-Matti Leppänen, Pipsa Saharinen, et al.

Biology of the TAM Receptors Greg Lemke
The Genesis of Tyrosine Phosphorylation Tony Hunter

Structure-Function Relationships of ErbB RTKs in the Plasma Membrane of Living Cells

Donna J. Arndt-Jovin, Michelle G. Botelho and Thomas M. Jovin

Receptor Tyrosine Kinases: Legacy of the First

Two Decades Joseph Schlessinger

The Role of Ryk and Ror Receptor Tyrosine Kinases in Wnt Signal Transduction Jennifer Green, Roel Nusse and Renée van Amerongen

Regulation of Receptor Tyrosine Kinase Ligand Processing Colin Adrain and Matthew Freeman

Molecular Mechanisms of SH2- and PTB-Domain-Containing Proteins in Receptor Tyrosine Kinase Signaling Melany J. Wagner, Melissa M. Stacey, Bernard A. Liu, et al.

Eph Receptor Signaling and Ephrins Erika M. Lisabeth, Giulia Falivelli and Elena B. Pasquale

Effects of Membrane Trafficking on Signaling by

Receptor Tyrosine Kinases Marta Miaczynska

For additional articles in this collection, see http://cshperspectives.cshlp.org/cgi/collection/

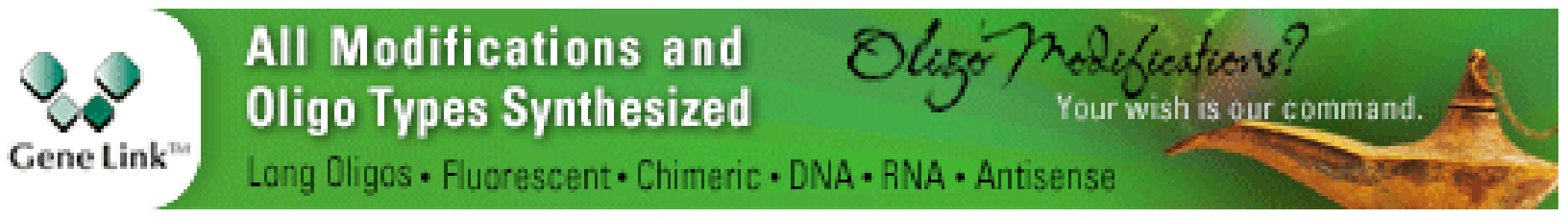


For additional articles in this collection, see http://cshperspectives.cshlp.org/cgi/collection/

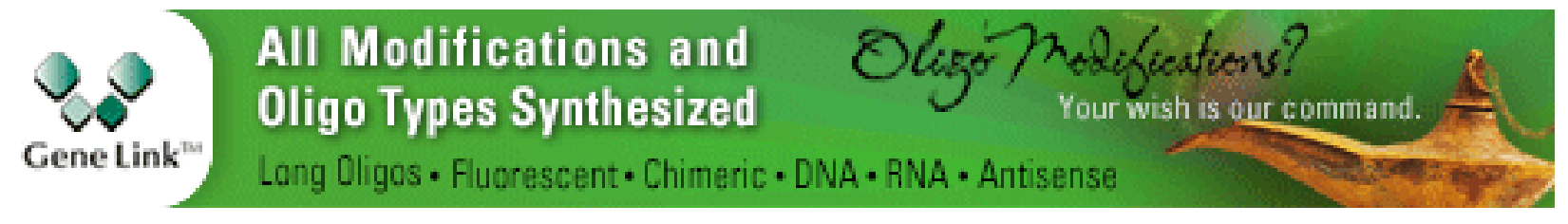

Copyright @ 2013 Cold Spring Harbor Laboratory Press; all rights reserved 